\title{
Manejo y reconstruccion microuuirúryica de extremidad inferior distal con colgajo SCIP libre
}

Managementand microsuryical reconstruction of distal lower limb with SCIP free flap

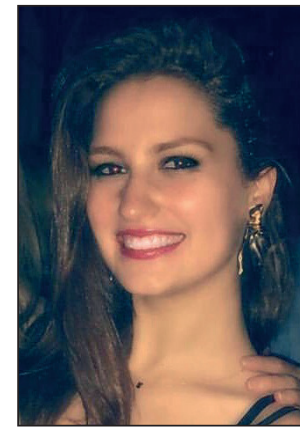

Malagón López P.

\section{Resumen}

Introducción y objetivo. En los últimos años han surgido nuevas técnicas de reconstrucción mediante supermicrocirugía con buenos resultados estéticos y funcionales, como el colgajo libre SCIP (Superficial Circumflex Iliac Artery Perforator), que permite realizar anastomosis a perforantes pudiendo conservar así los vasos principales de la extremidad.

El objetivo de este estudio es presentar nuestra experiencia en la reconstrucción de extremidad inferior distal con SCIP libre y su manejo perioperatorio.

Material y método. Nuestro estudio incluye 17 pacientes en los que llevamos a cabo reconstrucción mediante SCIP libre de extremidad inferior distal (pierna y pie) entre 2015 y 2017. Realizamos angioTAC preoperatorio para planificar la cirugía y valorar la necesidad de optimización vascular.

Resultados. Preferentemente hicimos anastomosis término-terminales a perforantes de la zona receptora. No fueron necesarias revisiones microquirúgicas inmediatas. La complicación postoperatoria más frecuente fue la congestión venosa (29.4\%). En todos los pacientes se logró la conservación de la extremidad y se mantuvo la capacidad de deambulación.

Conclusiones. el SCIP libre es una buena opción microquirúrgica para la reconstrucción de extremidad inferior distal, tanto estética como funcionalmente, ya que presenta escasas complicaciones y puede evitar la amputación. En base a nuestra experiencia inicial, recomendamos realizar angioTAC y valoración preoperatoria de optimización de la vascularización regional, cambios posturales, 2 anastomosis venosas siempre que sea posible y un manejo multidisciplinario.

Palabras clave SCIP, Extremidad inferior, Colgajo perforante, Colgajo libre.

\section{Nivel de evidencia científica Recibido [esta versión] Aceptado}

4c Terapéutico

Conflicto de intereses: Los autores declaran no tener ningún interés financiero relacionado con el contenido de este artículo. Financiación: No hubo fuentes externas de financiación para este trabajo.

Background and objetive. New reconstruction techniques have been described for last years with good aesthetic and functional results, such as the free SCIP flap (Superficial Circumflex Iliac Artery Perforator). Supermicrosurgery allows anastomoses to perforator vessels and therefore the main vessels of the limb can be preserved.

The aim of this study is to present our experience in the reconstruction of the distal lower extremity with free SCIP and its perioperative management

Methods. From 2015 to 2017, 17 patients who underwent a reconstruction of the distal lower extremity (leg and foot) by free SCIP flap were included. Preoperative CT-angiography was performed for the surgical planning and to assess the need for angioplasty.

Results. End-to-end anastomoses were made to perforator vessels of the recipient area. No immediate microsurgical revisions were needed. The most frequent postoperative complication was venous congestion (29.4\%). Limb salvage was achieved in all patients and walk ability was preserved.

Conclusions. The free SCIP flap is a good microsurgical option for the reconstruction of the distal lower extremity, both aesthetically and functionally, presents few complications and can avoid amputation. Based on our initial experience, we recommend performing a CT-angiography and a preoperative assessment of regional vascularization optimization, postural changes, 2 venous anastomoses whenever possible and multidisciplinary management.

Key words SCIP, Lower extremity, Perforator flaps, Free flaps.

\section{Level of evidence Received [this version] Accepted} 4c Therapeutic $20 \mathrm{May} / 2019$ 10 June/2019 


\section{Introducción}

La reconstrucción de la extremidad inferior distal es un reto quirúrgico, especialmente en aquellos casos que presentan alto riesgo de complicaciones como diabetes o vasculopatía avanzada. Están descritos varios tipos de colgajos locales y regionales, como el colgajo sural o el extensor de los dedos, que presentan sus indicaciones, ventajas e inconvenientes. ${ }^{(1-5)}$ Sin embargo, el principal problema reside en la falta de fiabilidad de su vascularización, especialmente en aquellos pacientes que ya presentan vasculopatía de base o pérdida de calidad de tejido por patologías crónicas de base o por el elevado grosor del colgajo que crea defectos de contorno.

En los últimos años han surgido nuevas técnicas de reconstrucción mediante supermicrocirugía, la cual permite aportar tejidos a distancia con buena calidad, alejados del lecho a reconstruir y realizar anastomosis a perforantes pudiendo conservar así los vasos principales de la extremidad. Además, presenta buenos resultados estéticos y funcionales. Entre estos colgajos encontramos el SCIP (Superficial Circumflex Iliac Artery Perforator, Perforante de la Arteria Circunfleja Superficial), descrito en 2004 por Koshima y que se diferencia del colgajo inguinal, primer colgajo libre descrito en 1973, en que está basado únicamente en una perforante de la arteria circunfleja iliaca superficial (SCIA). Sus principales ventajas son un tiempo de elevación del colgajo más corto, una baja morbilidad en la zona donante y que se trata de un colgajo fino que además permite un adelgazamiento aún mayor, ya sea directo o mediante disección suprafascial. Como desventajas, su pedículo es corto, de 1.5-4 cm, y los vasos presentan un pequeño calibre: el diámetro de SCIA (Superficial Circunflex Iliac Artery) es de $0.8-2 \mathrm{~mm}$, y sus perforantes del tercio medio y las venas superficiales son de $0.3-0.5 \mathrm{~mm} \cdot{ }^{(6-9)}$

El objetivo de este estudio es presentar nuestra experiencia en la reconstrucción de la extremidad inferior distal mediante el colgajo SCIP libre, así como el manejo perioperatorio.

\section{Material y método}

Incluimos en este estudio todos los pacientes intervenidos en el Hospital Germans Trias i Pujol de Badalona (Barcelona, España) para reconstrucción de miembro inferior distal (pierna y pie) mediante SCIP libre entre 2015 y 2017.

Recogimos los datos clínicos de los pacientes: edad, sexo, antecedentes patológicos de interés, el motivo de la reconstrucción, la técnica intraoperatoria, el tiempo de cirugía, las pruebas complementarias, la necesidad final de amputación, el resultado y las complicaciones postoperatorias.

\section{Manejo y técnica quirúrgica}

De forma preoperatoria solicitamos una prueba de imagen, preferiblemente angioTAC, para valorar el estado vascular e identificar y seleccionar las posibles perforantes que se emplearán en la reconstrucción. En aquellos casos en los que se objetivó una estenosis arterial significativa se realizó repermeabilización mediante angioplastia de forma preoperatoria para optimizar el flujo distal.

En el preoperatorio inmediato, marcamos las posibles perforantes tanto en la zona donante como en la receptora mediante doppler.

Durante la intervención trabajan 2 equipos: 1 o 2 cirujanos en la zona donante para elevar el colgajo y 1 o 2 en la zona receptora para el desbridamiento o exéresis tumoral y la búsqueda de perforantes mediante el uso de lupas o microscopio.

Al tratarse de vasos de pequeño calibre, como norma general primero identificamos las perforantes receptoras pulsátiles y las seccionamos para comprobar que presentan un flujo correcto, y en segundo lugar realizamos la autonomización del colgajo. Posteriormente llevamos a cabo la dilatación de los vasos y la infiltración tópica con heparina diluida. La anastomosis la realizamos término-terminal, a ser posible perforante-perforante, con 1 o 2 venas con monofilamento irreabsorbible de 10/0 - 11/0. Cerramos la zona donante de forma directa mediante puntos subcutáneos de vicryl 3/0 e intradérmica con monocryl 3/0.

\section{Resultados}

Durante el periodo señalado realizamos un total de 17 reconstrucciones de miembro inferior distal (pierna y pie) mediante SCIP libre: 10 úlceras en pie diabético, 3 melanomas, 3 traumatismos y 1 úlcera de origen vascular (Fig. 1). La edad media de los pacientes intervenidos fue de 58 años (rango 26-79 años). Cuatro de los pacientes incluidos fueron mujeres $(23.5 \%)$ y 13 fueron hombres (76.4\%). El tamaño medio de los colgajos fue de $9.5 \mathrm{x}$ $5.9 \mathrm{~cm}$ (rango, 5-20 x 4-8 cm). En la Tabla I recogemos otros datos clínicos de interés.

En todos los casos llevamos a cabo angioTAC de forma preoperatoria objetivando estenosis significativa $\mathrm{u}$ obliteración completa en al menos 1 vaso de la extremidad afectada en 10 pacientes (el 100\% de los pacientes con úlceras diabéticas) (Fig. 2). De todos ellos, 6 presentaban afectación de los 3 vasos principales de la pierna (peronea, tibial anterior y posterior) y a 7 pacientes se les realizó angioplastia preoperatoria.

El tiempo medio de microcirugía fue de $230 \mathrm{~min}$ (160-310 min). 


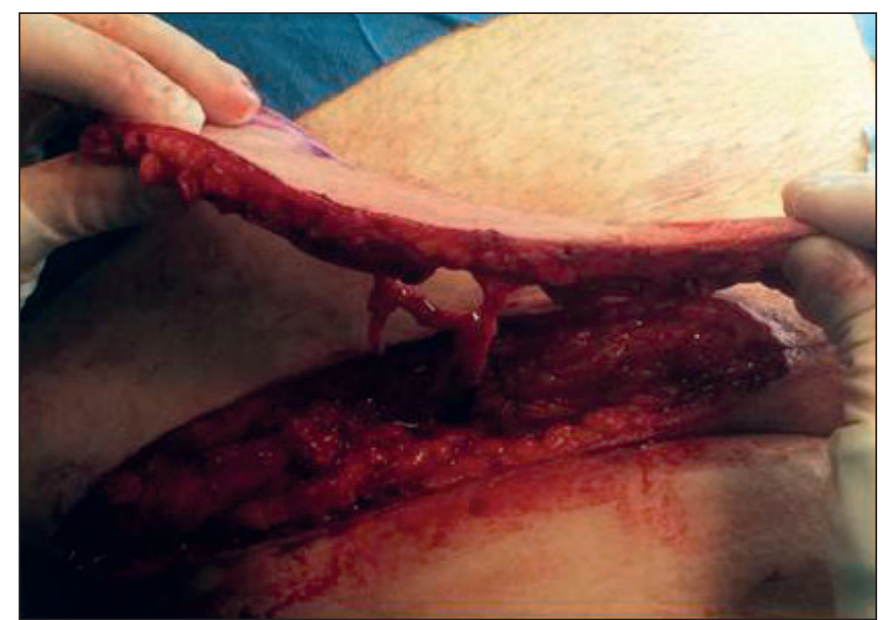

Figura 1. Disección del colgajo SCIP en zona donante. Se observa el fino grosor del colgajo que permite mejores resultados en el contorno de la extremidad inferior distal reconstruida, especialmente, en el dorso de pie.

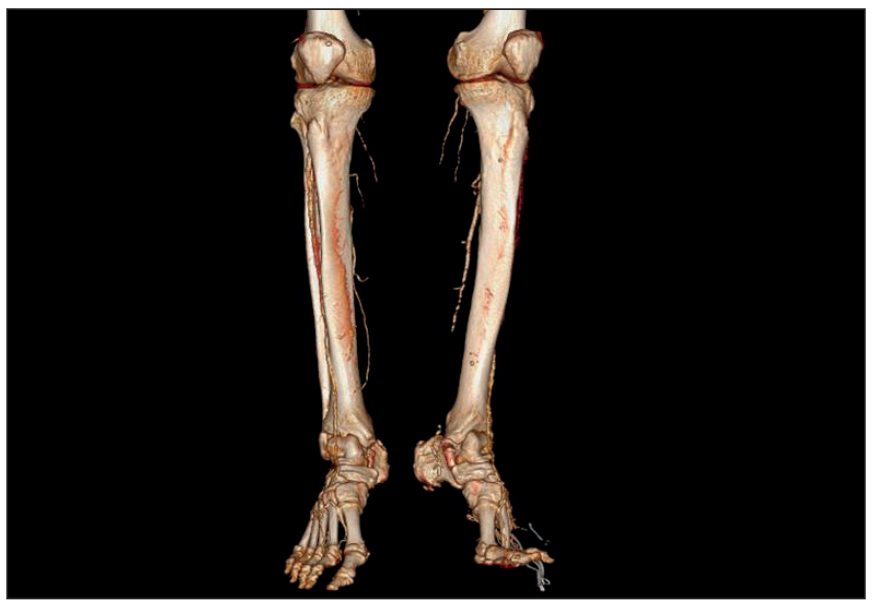

Figura 2. Imagen de reconstrucción 3D de TAC con contraste endovenoso de paciente con úlcera diabética izquierda que muestra estenosis significativas y críticas en arteria y troncos principales, con colateralidad arterial distal efectiva y menor afectación ateromatosa de la arteria pedia.
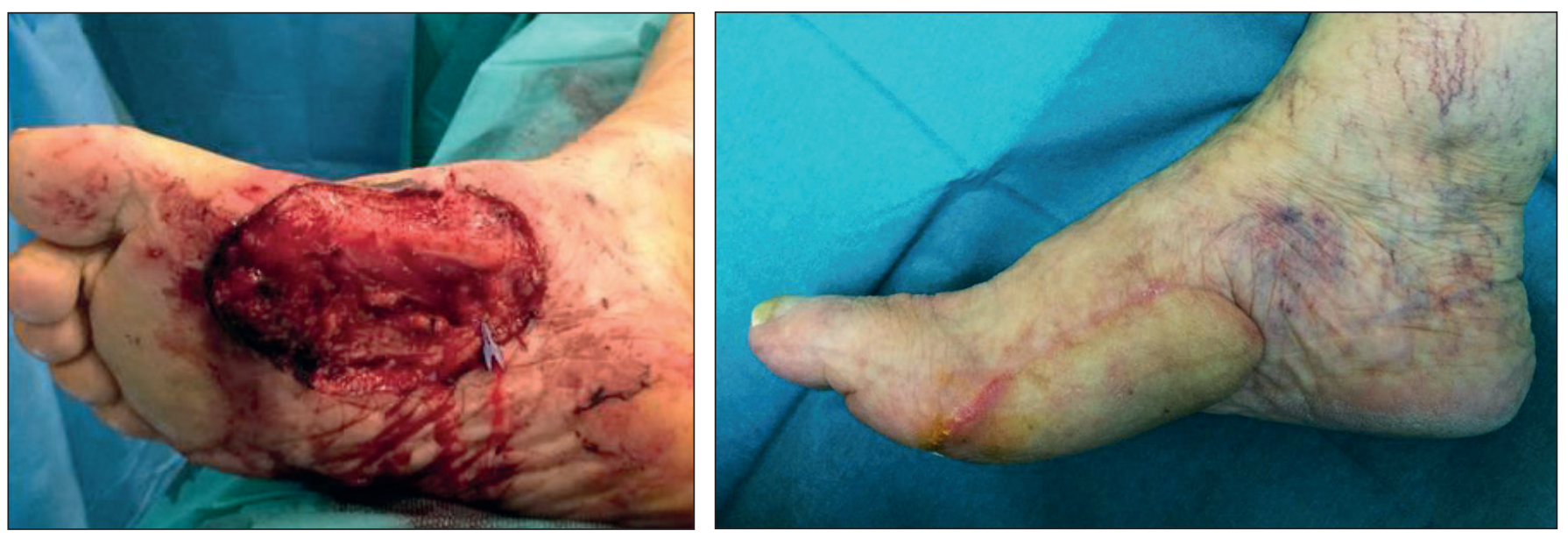

Figuras 3 y 4 . Mujer de 75 años diagnosticada de melanoma plantar de pie izquierdo, Breslow $1.3 \mathrm{~mm}$ de $2 \times 1.5 \mathrm{~cm}$. Exéresis con márgenes de $2 \mathrm{~cm}$ y cobertura mediante SCIP libre anastomosado a perforantes plantares (1 arteria, 1 vena). Imagen intraoperatoria y resultado a los 3 meses de la intervención.
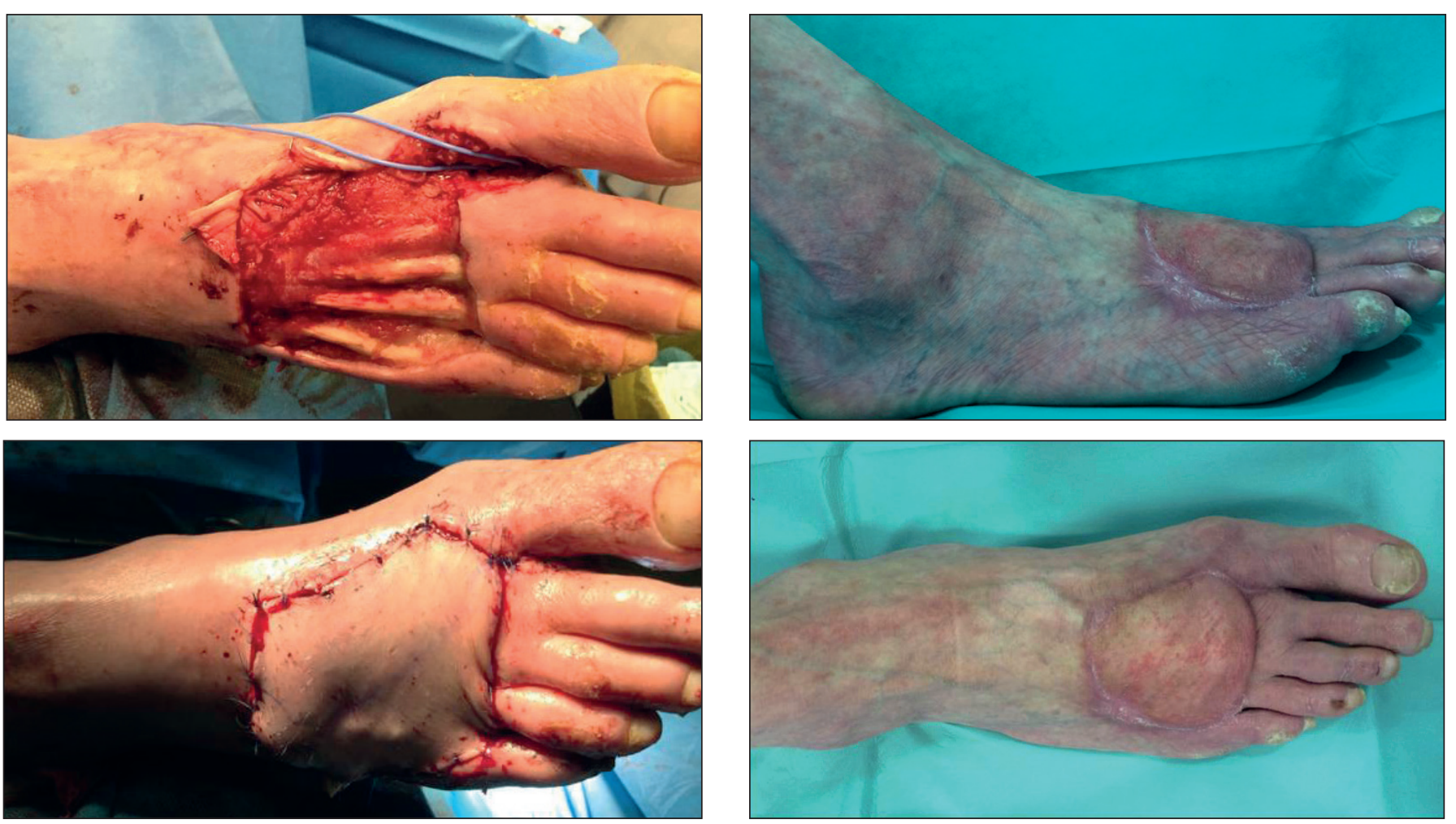

Figuras 5-8. Varón de 40 años fumador que sufre traumatismo accidental con pérdida de sustancia de dorso de pie y exposición de tendones extensores. Tras el desbridamiento, se realiza cobertura mediante SCIP libre con anastomosis de 1 arteria y 2 venas (arteria y vena perforantes y vena superficial accesoria). Imágenes intraoperatorias y resultado a los 4 meses de la intervención. 
Tabla I. Datos clínicos de los pacientes incluidos en el estudio.

\begin{tabular}{|l|c|}
\hline & Prevalencia (n, \%) \\
\hline Hipertensión arterial & $5,29.4 \%$ \\
\hline Diabetes mellitus & $12,70.5 \%$ \\
\hline Dislipemia & $3,17.6 \%$ \\
\hline Obesidad (IMC* $>30)$ & $4,23.5 \%$ \\
\hline Fumador & $4,23.5 \%$ \\
\hline Causa de la reconstrucción: & \\
Pie diabético & $10,58.8 \%$ \\
Tumoral & $3,17.6 \%$ \\
Traumática & $3,17.6 \%$ \\
Úlcera vascular & $1,5.8 \%$ \\
\hline
\end{tabular}

*IMC= Índice de masa corporal

Como complicaciones postquirúrgicas se produjeron 5 casos de congestión venosa tardía (a partir del $5^{\circ}$ día de postoperatorio) que requirieron tratamiento quirúrgico: en todos ellos se produjo necrosis cutánea parcial o total con buen aspecto del plano subcutáneo del colgajo, por lo que practicamos cobertura con autoinjerto de piel parcial laminar con correcta viabilidad y funcionalidad final.

En todos los casos logramos la conservación de la extremidad y todos iniciaron rehabilitación de forma precoz (Fig. 3-7). Antes del alta hospitalaria todos los pacientes fueron valorados por el Servicio de Rehabilitación del hospital y se programó una visita en consultas externas 1 mes después de la intervención quirúrgica. El tiempo medio de inicio de la deambulación fué de 3 semanas (rango de 2-7 semanas).

\section{Discusión}

La reconstrucción de miembro inferior distal puede resultar un reto para el cirujano a la hora de elegir el colgajo que mejor se adapte a las necesidades de cada caso, por lo que será necesario analizar las ventajas e inconvenientes de cada colgajo en cada paciente.

El SCIP puede ser una buena opción reconstructiva, con una escasa morbilidad y una rápida recuperación de la zona donante y que puede llegar a evitar la necesidad de amputación de la extremidad. ${ }^{(7,8,10)}$ Además, es un colgajo muy versátil que permite la incorporación de otros tejidos con relativa facilidad (fascia, tejido óseo, nervio, ganglios linfáticos,) por lo que se amplía el rango de situaciones en las que puede ser de utilidad. ${ }^{(11,12)}$ Sin embargo, hay que tener en cuenta que su disección y anastomosis pueden ser técnicamente complicadas, puesto que el calibre de los vasos es pequeño y el pedículo presenta una escasa longitud.
Al compararlo con otras opciones microquirúrgicas, como el colgajo ALT (Anterolateral Thigh, anterolateral de muslo), presenta la ventaja de ser un colgajo más delgado con mejor capacidad de adaptación a la zona a reconstruir, especialmente en el dorso del pie, ${ }^{(12-14)}$ la menor presencia de pelo y ocasiona una cicatriz más estética en la zona donante.

Si lo comparamos con el colgajo radial, las principales ventajas radican en la menor morbilidad en la zona donante y la conservación de la arteria. Sin embargo, una vez más hay que tener en cuenta la menor longitud y calibre del pedículo.

Por otro lado, es importante seleccionar los vasos receptores. En los últimos años ha aparecido un nuevo concepto que defiende la realización de la anastomosis a vasos más distales en lugar de a los troncos principales de la pierna. En 1959 Goldenberg describió una mayor alteración en los vasos distales en los pacientes diabéticos, sobre todo oclusiones arteriolares, que parecían ser la causa de la mayor incidencia de úlceras isquémicas distales en estos pacientes. ${ }^{(15)}$ Sin embargo, estudios posteriores han fracasado en la demostración de dicha oclusión arteriolar o de la proliferación endotelial. A nivel capilar sí se ha documentado un engrosamiento en la membrana basal capilar, pero tampoco oclusión capilar. ${ }^{(16)}$ En otros estudios también se ha encontrado una mayor incidencia de alteraciones ateroscleróticas en las arterias tibiales y peroneas en los pacientes diabéticos, con mucha menor afectación de las estructuras vasculares distales. ${ }^{(17-19)}$

Por tanto, es posible que se consiga un mejor resultado si la anastomosis del colgajo se realiza a vasos más distales, surgiendo así la reconstrucción de extremidad inferior a través de perforantes. ${ }^{(20-25)}$ Por este motivo los vasos receptores de primera elección por parte de los autores son las perforantes distales, en caso de que haya disponibilidad de estos. Aunque de forma poco frecuente, especialmente en pacientes que presentan inflamación de tejidos blandos de forma crónica e importante fibrosis, no se consigue localizar perforantes distales pulsátiles de forma intraoperatoria. En estos casos, optamos por realizar la anastomosis a la arteria pedia y, en tercer lugar, a troncos principales.

Uno de los problemas más importantes que hemos encontrado en las reconstrucciones mediante el colgajo SCIP libre en miembro inferior distal ha sido la congestión venosa: en total, 5 casos de 17 (29.4\%) requirieron injerto de piel. Debemos tener en cuenta que manejamos vasos de calibre reducido que son muy sensibles al vasoespasmo si no se realiza una técnica cuidadosa, especialmente durante la disección de los vasos receptores en pacientes diabéticos y vasculópatas. Basándonos en 
nuestra experiencia, consideramos conveniente realizar 2 anastomosis venosas siempre que sea posible y modificar cuidadosamente los cambios posturales de la extremidad durante los primeros días del postoperatorio debido a la baja capacidad de adaptación del colgajo a los cambios hemodinámicos. En el caso de que el colgajo presente anastomosis permeables pero palidez cutánea, se indica descenso de la extremidad $10-20^{\circ}$. Si a pesar de ello persiste la clínica, se indica nuevo descenso de $10-20^{\circ}$ más. Por el contrario, si el colgajo inicia signos de edema o congestión venosa se indica elevación del miembro $30^{\circ}$ con revaloración clínica a la hora. Según nuestra experiencia, el edema y la congestión venosa del colgajo son más frecuentes que la isquemia y son especialmente habituales entre el segundo y el quinto día de postoperatorio. Por este motivo es importante la observación clínica cercana del paciente durante la primera semana.

Otro aspecto a destacar es la importancia de un manejo multidisciplinario para conseguir un resultado óptimo y funcional en estos pacientes, especialmente en aquellos que presentan factores de riesgo como vasculopatía y diabetes. ${ }^{(26)}$ En nuestro hospital existe un estrecho contacto entre los Servicios de Cirugía Plástica, Cirugía Vascular, Traumatología, Infecciosas, Endocrinología, Radiología y Rehabilitación, que de forma conjunta valoran los casos complejos de reconstrucción de miembro inferior tanto en el diagnóstico como en la toma de decisiones de tratamiento conservador o quirúrgico y en el seguimiento de los pacientes. La colaboración entre los equipos es fundamental para conseguir una optimización preoperatoria que mejore la vascularización distal de la extremidad, el estado general del paciente, una reconstrucción que permita ser funcional y una rehabilitación precoz, que harán posible la mejora en su calidad de vida .

\section{Conclusiones}

En nuestra serie de casos, la reconstrucción microquirúrgica de extremidad inferior distal mediante el colgajo SCIP fue una buena opción, tanto estética como funcional, que evitó la necesidad de amputación con escasas complicaciones.

Las complicaciones quirúrgicas más frecuentes que encontramos estuvieron relacionadas con el edema y la congestión venosa, especialmente durante los primeros 5 días de postoperatorio.

Basándonos en nuestra experiencia inicial, recomendamos realizar un angioTAC y una valoración preoperatoria de optimización de la vascularización regional, 2 anastomosis venosas siempre que sea posible y un manejo multidisciplinario.
Dirección del autor

Dra. Paloma Malagón López

Hospital Germans Trias i Pujol

Servicio de Cirugía Plástica

Carretera de Canyet s/n

08916

Badalona, Barcelona, España

Correo electrónico: pal.malagon@gmail.com

Bibliografia

1. Benito-Duque P. Modificaciones del colgajo sural invertido para aumentar su viabilidad en reconstrucción de grandes defectos del pie. Cir. plást. iberolatinoam., 2018;44(1): 65-71.

2. Peralta-Jerezano A., Carpinteyro-Espín U. Colgajo de abductor digiti minimi, una opción vigente en la reconstrucción de tejidos blandos del pie. Cir. plást. iberolatinoam., 2016;42(3): 293-297.

3. Mendieta-Espinosa M. Colgajos de perforante en hélice para reconstrucción de defectos de tercio medio y distal de pierna. Cir. plást. iberolatinoam., 2017;.43(1): 87-96.

4. Mazarrasa-Marazuela B., Delgado-Giraldo P.A., Benito-Duque P. Colgajo fasciocutáneo safeno interno desdoblado. Cir. plást. iberolatinoam., 2014;40(2): 179-184.

5. Fonseca Portilla G., Vargas Naranjo S. Reconstrucción de pierna con colgajo venofasciocutáneo de safena menor: Hospital San Juan de Dios de Costa Rica, 2004-2009. Cir. plást. iberolatinoam., 2012;38(2):137-144.

6. Fu-Chan Wei, Samir Mardini. Flaps and Reconstructive Surgery. 2nd edition. Part 4. Section E. Chapter 43. Pp. 478-487.

7. Isao K, Yuzabo N, Tetsuya T et al. Superficial Circunflex Iliac Artery Perforator Flap for Reconstruction of Limb Defects. Plast Reconstr Surg. 2004;113(1):233-240.

8. Jong Hwan K, Kyu Nam K, Chi Sun Y. Reconstruction of Moderate-Sized Distal Limb Defects using a Superthin Superficial Circumflex Iliac Artery Perforator Flap. J Reconstr Microsurg. 2015;31:631-635.

9. Goh TL, Park SW, Cho JY, Choi JW, Hong JP. The search for the ideal thin skin flap: superficial circumflex iliac artery perforator flap-a review of 210 cases. Plast Reconstr Surg. 2015;135(2):592-601.

10. Oh TS, Lee HS, Hong JP. Diabetic foot reconstruction using free flaps increases 5-year-survival rate. J Plast Reconstr Aesthet Surg. 2013;66(2):243-250.

11. Hong JP, Sun SH, Ben-Nakhi M. Modified superficial circumflex iliac artery perforator flap and supermicrosurgery technique for lower extremity reconstruction: a new approach for moderate-sized defects. Ann Plast Surg. 2013;71(4):380-383.

12. Lida T. Superficial Circumflex Iliac Perforator (SCIP) Flap: Variations of the SCIP Flap and Their Clinical Applications. $J$ Reconstr Microsurg. 2014;30:505-508.

13. Myung Y, Yim S, Kim BK. A comparison of axial circumference between superficial circumflex iliac artery perforator flap and other workhorse flaps in dorsal foot reconstruction. $J$ Plast Surg Hand Surg. 2017;51(6):381-386.

14. Goldenberg S, Alex M, Joshi RA, Blumenthal HT. Nonatheromatous peripheral vascular disease of the lower extremity in diabetes mellitus. Diabetes. 1959;8(4):261-273.

15. Banson BB, Lacy PE. Diabetic microangiopathy in human toes, with emphasis on the ultrastructural change in dermal capillaries. Am. J. Path. 1964;45:41

16. Kallio M, Vikatmaa P, Kantonen I, et al. Strategies for Free Flap Transfer and Revascularisation with Long-term Outcome in 
the Treatment of Large Diabetic Foot Lesions. Eur J Vasc Endovasc Surg. 2015;50:223-230.

17. Conrad MC. Large and small artery occlusion in diabetics and nondiabetics with severe vascular disease. Circulation. 1967; 36: 83-91.

18. Barner HB, Kaiser GC, Willman VL. Blood flow in the diabetic leg. Circulation. 1971; 43: 391-394.

19. LoGerfo FW, Coffman JD. Current concepts. Vascular and microvascular disease of the foot in diabetes. Implications for foot care. N Engl J Med. 1984; 311: 1615-1619.

20. Hong JP. The Use of Supermicrosurgery in Lower Extremity Reconstruction: The Next Step in Evolution. Plast Reconstr Surg. 2009;123:230-235.

21. Suh HS, Oh TS, Lee HS, Lee SH, Cho YP, Park JR, Hong JP. A New Approach for Reconstruction of Diabetic Foot Wounds Using the Angiosome and Supermicrosurgery Concept. Plast Reconstr Surg. 2016;138:702-709.
22. Suh HS, Oh TS, Hong JP. Innovations in diabetic foot reconstruction using supermicrosurgery. Diabetes Metab Res Rev. 2016;32 Suppl 1:275-280.

23. Seo BF, Choi JY, Han HH, et al. Perforators as recipients for free flap reconstruction of the inguinal and perineal region. $\mathrm{Mi}$ crosurgery. 2015;35(8):627-633.

24. Kim KN, Hong JP, Park SW, et al. Overcoming the Obstacles of the Ilizarov Device in Extremity Reconstruction: Usefulness of the Perforator as the Recipient Vessel. J Reconstr Microsurg. 2015;31:420-425.

25. Hong JP, Tae Suk Oh. An Algorithm for Limb Salvage for Diabetic Foot Ulcers. Clin Plastic Surg. 2012;39:341-352.

26. Wraight PR, Lawrence SM, Campbell DA, et al. Creation of a multidisciplinary, evidence based, clinical guideline for the assessment, investigation and management of acute diabetes related foot complications. Diabet Med. 2005; 22:127-136.

\title{
Comentario al artículo “Manejo y reconstrucción microquirúrgica de extremidad inferior distal con colgajo SEIP libre"
}

\author{
Pablo BENITO DUQUE \\ Cirujano Plástico, Jefe del Servicio de Cirugía Plástica del Hospital Universitario Ramón y Cajal, Madrid, España
}

He leído con interés el trabajo presentado por Malagón y col. sobre la utilidad del colgajo SCIP para la reconstrucción de la extremidad inferior. La serie de 17 pacientes tratados con dicha técnica, así como los resultados obtenidos, sus complicaciones y la discusión, nos ayudan a conocer mejor las posibilidades que puede ofrecer en comparación con otras técnicas más habituales, tanto microquirúrgicas como regionales.

Coincido en la importancia que dan los autores al manejo multidisciplinar, especialmente indicado en vasculopatía y diabetes. Indispensable para disminuir complicaciones y como explican en el artículo, para permitir intervenir pacientes que inicialmente no serian candidatos a este tipo de colgajo.

No obstante, al analizar la serie, llama la atención el elevado número de pacientes diabéticos $(58.8 \%)$, que a pesar de la colaboración multidisciplinar que acertadamente realizan los autores (angioplastia), creo puede ser un factor de sesgo inevitable.

Los autores señalan en la Discusión como uno de los problemas más importantes la congestión venosa, llegando en la serie presentada a requerir reintervención con injerto el 29.4\%. Considero que es una cifra elevada, y debe hacer recapacitar principalmente en los criterios de inclusión que se deben seguir en el algoritmo de elección del colgajo en relación a las patologías acompañantes de cada paciente. Dicho problema se asocia a otros colgajos como el sural de flujo reverso y ha merecido numerosas publicaciones. ${ }^{(1,2)}$ La necesidad de la aplicación de un injerto de piel en un pie diabético puede llegar a comprometer el resultado final, puesto que se trata de una zona que estará sometida a presión y roce. Sin olvidar la posibilidad de que coexista neuropatía periférica ( $50 \%$ de los diabéticos). También habría sido interesante conocer las zonas reconstruidas en el pie, especificando si corresponden a zonas plantares, dorsales o de talón, pues el periodo de seguimiento y posibles complicaciones varían.

El trabajo presentado significa un paso más en el perfeccionamiento técnico de la Cirugía Reconstructiva con el objetivo de minimizar la morbilidad tanto en la zona receptora como en la extremidad a reconstruir y animará a aquellos cirujanos implicados en esta área a considerarlo entre sus posibilidades. Felicito a los autores por su pericia en este tipo de colgajo, con una tasa de supervivencia del $100 \%$ a pesar de abordar un elevado porcentaje de pacientes con vasos periféricos comprometidos.

\section{Bibliografía}

1. Parrett B.M., Pribaz J.J., Matros E. y col. Risk analysis for the reverse sural fasciocutaneous flap in distal leg reconstruction. Plast Reconstr Surg 2009;123:1499-1504.

2. Foran P., Schreiber J., Christy M.R. y col. The modified reverse sural artery flap lower extremity reconstruction. J Trauma 2008;64:139-143. 


\section{Respuesta al comentario de Dr. Pablo Benito Duque}

\section{Paloma MALAGÓN LÓPEZ}

En primer lugar, queríamos agradecer al Dr. Benito Duque, Jefe del Servicio de Cirugía Plástica del H.U. Ramón y Cajal de Madrid, España, por el reconocimiento de nuestro trabajo y sus interesantes comentarios. También agradecemos a la Dra. Vaquero Pérez, directora de la Revista Cirugía Plástica Ibero-Latinoamericana, la oportunidad de poder compartir nuestra experiencia y aprendizaje acerca de las lesiones de extremidad inferior distal.

El manejo y tratamiento de este tipo de defectos constituye todo un reto debido a su elevada complejidad, sobre todo y como bien señala el Dr. Benito por la elevada tasa de diabetes avanzada, vasculopatías y otras comorbilidades asociadas. La mayoría de los casos que hemos presentado habrían sido candidatos a amputación si la reconstrucción microquirúrgica no hubiera sido posible o hubiera fracasado. Sin embargo, es fundamental tratar de preservar la extremidad precisamente cuando existen estas patologías asociadas, puesto que estos pacientes presentan un riesgo añadido de padecer una nueva úlcera, tanto en la extremidad reconstruida como en la contralateral y, por tanto, mayor riesgo de una segunda amputación. ${ }^{(1,2)}$ En la serie presentada, mediante el manejo perioperatorio descrito y la realización de una reconstrucción microquirúrgica con colgajo SCIP libre, se logró la preservación de la extremidad y su funcionalidad en todos los casos.

Una de las dificultades que encontramos es que este tipo de manejo y reconstrucción microquirúrgica requieren una curva de aprendizaje. Al inicio de la introducción de esta técnica en nuestro centro, también nos llamó la atención la elevada tasa de congestión venosa que presentaban los colgajos. Por esta razón, se instauró la realización de dos anastomosis venosas por protocolo y con ello disminuyó la aparición de congestión y mejoraron los resultados finales. A pesar de nuestra corta experiencia, creemos que el colgajo SCIP es un colgajo muy útil para este tipo de defectos en extremidad inferior, ya que por el diámetro del pedículo es posible realizar una anastomosis a vasos más distales, conservando así los troncos arteriales principales de la pierna, por su fácil adaptación a los defectos en esta área anatómica y por la mínima morbilidad de la zona donante.

Coincidimos plenamente con el Dr. Benito en que no solo la patología de base es importante para el tratamiento de este tipo de lesiones, sino también tener en cuenta la zona de la extremidad que está afectada y su función. En nuestra serie de casos se incluyeron todas las regiones del pie y tobillo, incluyendo las zonas de carga: región plantar para cobertura de tercio distal de metatarsianos, región plantar medial, dorso de pie, talón, maleolos y tercio pretibial distal. También coincidimos en que debido a que las áreas reconstruidas están sometidas a fuerzas de fricción y/o presión durante la deambulación, es preferible una reconstrucción mediante colgajo en lugar de un injerto. Al lograr disminuir la tasa de congestión venosa mediante la realización de dos anastomosis venosas de forma rutinaria también se ha reducido la necesidad de injertos en un segundo tiempo, por lo que consideramos que el colgajo SCIP es óptimo para la reconstrucción de extremidad inferior distal.

Finalmente, queríamos agradecer nuevamente su interés y sus palabras. Para nosotros es un privilegio poder compartir este estudio y esperamos que sea de utilidad y que permita mejorar el manejo y la reconstrucción de este tipo de defectos tan complejos, con el objetivo final de reducir la tasa final de amputación y mejorar la calidad de vida de los pacientes.

Bibliografia

1. Miller W., Berg C., Wilson ML. et al. Risk Factors for Belowthe-Knee Amputation in Diabetic Foot Osteomyelitis After Minor Amputation. J Am Podiatr Med Assoc. 2019;109(2):91-97.

2. Armstrong DG., Boulton AJM., Bus SA. Diabetic Foot Ulcers and Their Recurrence. N Engl J Med. 2017; 376(24):2367-2375. 
